\title{
New method for fitting coefficients in standard model effective theory
}

\author{
Geoffrey T. Bodwin $\oplus^{1, *}$ and Hee Sok Chung $\odot^{2,3, \dagger}$ \\ ${ }^{1}$ High Energy Physics Division, Argonne National Laboratory, Argonne, Illinois 60439, USA \\ ${ }^{2}$ Physik-Department, Technische Universität München, James-Franck-Str. 1, 85748 Garching, Germany \\ ${ }^{3}$ Excellence Cluster ORIGINS, Boltzmannstrasse 2, D-85748 Garching, Germany
}

(Received 24 January 2020; accepted 5 June 2020; published 30 June 2020)

\begin{abstract}
We present an alternative method for carrying out a principal-component analysis of Wilson coefficients in standard model effective field theory (SMEFT). The method is based on singular-value decomposition (SVD). The SVD method provides information about the sensitivity of experimental observables to physics beyond the standard model that is not accessible in the Fisher-information method. In principle, the SVD method can also have computational advantages over diagonalization of the Fisher information matrix. We demonstrate the SVD method by applying it to the dimension-6 coefficients for the process of top-quark decay to a $b$ quark and a $W$ boson and use this example to illustrate some pitfalls in widely used fitting procedures. We also outline an iterative procedure for applying the SVD method to dimension-8 SMEFT coefficients.
\end{abstract}

DOI: 10.1103/PhysRevD.101.115039

\section{INTRODUCTION}

In recent years, standard model effective theory (SMEFT) [1-4] has been a focus of activity in both the theoretical and experimental particle-physics communities. SMEFT has been advocated as a means to quantify systematically deviations of the global set of experimental measurements from the predictions of the standard model. To this end, a number of efforts have been undertaken to perform global fits of the Wilson coefficients of SMEFT operators. Some examples of global fits are contained in Refs. [5-19].

Several difficulties can arise in using data to constrain coefficients in SMEFT. First, there are many operators in SMEFT (59 in dimension 6) and potentially many data points that could be used in fitting the coefficients of these operators. Second, theoretical expressions for the SMEFT contributions to a given set of experimental observables can contain "flat directions" (or nearly flat directions) in the space of SMEFT coefficients, that is, directions for which the observables are insensitive to the values of SMEFT coefficients. Third, for analyses involving a limited sector of observables, there may be fewer observables than

\footnotetext{
gtb@anl.gov

heesok.chung@tum.de
}

Published by the American Physical Society under the terms of the Creative Commons Attribution 4.0 International license. Further distribution of this work must maintain attribution to the author(s) and the published article's title, journal citation, and DOI. Funded by SCOAP.
SMEFT coefficients. This situation will necessarily result in the existence of exactly flat directions.

All of these difficulties can pose computational problems in fitting SMEFT coefficients to data. A global fit may be computationally challenging because standard methods for carrying out fits may bog down when the number of observables and coefficients is large. When the number of coefficients is greater than the number of observables and/ or there are flat directions, methods of fitting that minimize $\chi^{2}$ numerically may not converge reliably. In addition to these technical issues, there is also an issue of principle: When there are more coefficients than observables and/or flat directions, the uncertainties in the coefficients can be highly correlated, and bounds on values of individual SMEFT coefficients may be very misleading.

This last issue of principle can be addressed by carrying out a principal-component analysis (PCA) of the SMEFT coefficients. One way to do this is by finding the eigenvalues and eigenvectors of the Fisher information matrix, which, in the case of Gaussian statistics, is the inverse of the correlation matrix [20-25]. Since the Fisher matrix is nonsingular, diagonalization of the Fisher matrix evades the computational problems that arise, when there are flat directions, in minimizing $\chi^{2}$ numerically.

Another approach is to regularize $\chi^{2}$, so that it is nonsingular and can be minimized by numerical methods [26]. In this approach, the regulator could potentially introduce biases into the fit.

In this paper, we present an alternative method for carrying out the PCA of the SMEFT coefficients that is based on singular-value decomposition (SVD). The SVD method has the same advantages as the diagonalization of 
the Fisher matrix in evading the computational problems that can arise in numerical minimization of $\chi^{2}$. In addition, the SVD method provides information about the sensitivity of experimental observables to physics beyond the standard model that is not accessible in the Fisher-information method.

There may also be algorithmic advantages in using SVD. Many well developed numerical methods exist both for diagonalization and for SVD. However, because the Fisher matrix is quadratic in the matrix to which SVD is applied, its condition number is the square of the condition number for the SVD matrix, and so, the precision may be worse for diagonalization of the Fisher matrix than for SVD [27]. Furthermore, if the number of SMEFT coeffients is much larger than the number of observables, then the matrix that is analyzed in the SVD approach is much smaller than the Fisher matrix, and so the computation time may be smaller in the SVD approach. It remains to be seen whether these advantages will be significant in extensive and/or iterated global fits of SVD coefficients.

We demonstrate the SVD method by applying it to a restricted class of observables that appear in decay of the top quark to a $b$ quark and a $W$ boson. ${ }^{1}$ This example allows us to show how the SVD method can be used to deal with correlated theoretical and experimental errors and with the difficulties of flat directions.

In fits of SMEFT coefficients in the literature, two methods that are often employed are (1) fits in which all of the SMEFT coefficients but one are set to zero and (2) fits in which all of the SMEFT coefficients but one are marginalized (profiled). It has been emphasized in Ref. [20] that both of these approaches are misleading and are obviated by PCA. In this paper, we demonstrate in explicit examples involving the SMEFT coefficients in the topquark sector, that approach (1) leads to overly optimistic constraints on the SMEFT coefficients, while approach (2) leads to overly pessimistic constraints on the SMEFT coefficients. Our ten-coefficient PCA results for the topquark-decay SMEFT coefficients should be considered to supersede the one-coefficient fit results in Ref. [31], in which approach (1) was used, and the two-coefficient fits in Ref. [31], which do not account completely for the high degree of correlation between the uncertainties in the coefficients.

While we have not specifically demonstrated the utility of the SVD method for a situation in which there is a large number of observables and a large number of SMEFT coefficients, we are confident that it would work reliably and efficiently in such a situation because of experience with an application of SVD, in a different context, that involved the fitting of thousands of data points with hundreds of coefficients [32].

\footnotetext{
${ }^{1}$ We note that global fits of SMEFT coefficients in the topquark sector have been carried out in Refs. [17,28-30].
}

The SVD method that we present is based on a Gaussian uncertainty analysis. While this, of course, is not completely general, it should prove to be adequate at least for initial exploratory studies of the bounds on SMEFT coefficients. The SVD method also requires that the observables depend linearly on the SMEFT coefficients. This is the case in a computation at leading order in the effective-field-theory expansion. As we will describe later, the method can also be used iteratively to carry out a PCA of the SMEFT coefficients in the case in which higher-order contributions in the effective-field-theory expansion are considered-provided that the expansion itself converges.

The remainder of this paper is organized as follows. In Sec. II, we outline the basics of SVD and present a method for using SVD to carry out analyses of SMEFT coefficients. Section III contains an illustration of the use of SVD analysis in top-quark decay to a $b$ quark and a $W$ boson. Here, we present examples of fits involving flat directions and various numbers of SMEFT coefficients, and we contrast the results from the PCA with those from the traditional fitting approaches (1) and (2) that are mentioned above. In Sec. IV, we discuss the extension of the SVD approach to situations in which the theoretical expressions for the observables depend non-linearly on the SMEFT coefficients. Finally, in Sec. V, we summarize our results.

\section{SINGULAR-VALUE DECOMPOSITION METHOD}

\section{A. Singular-value decomposition}

The singular-value-decomposition theorem states that an $m \times n$ matrix $M$ that contains either real or complex entries can always be decomposed as [33]

$$
M=U W V^{\dagger},
$$

where $U$ is an $m \times m$ unitary matrix, $V$ is an $n \times n$ unitary matrix, $W$ is an $m \times n$ diagonal matrix with nonnegative real numbers on the diagonals, and $\dagger$ denotes the Hermitian conjugate (complex conjugate transpose). The diagonal entries of $W$ are called the singular values. The matrix $W$ is unique, but the matrices $U$ and $V$ are not. If $M$ is a square matrix, then $U$ and $V$ are unique, up to phases that multiply each row of $V^{\dagger}$ and corresponding inverse phases that multiply each column of $U$. If $M$ is not a square matrix, additional ambiguities in $U$ and $V$ can arise. Efficient computer codes exist for carrying out the SVD decomposition of large matrices numerically. See, for example, Refs. [34-37].

SVD has the important property that it can be used to solve the linear least-squares problem, as we will now explain. Suppose that $M$ is an $m \times n$ matrix, $C$ is an $n$-dimensional column vector, and $O$ is an $m$-dimensional column vector. Further suppose that we wish to minimize 


$$
(M C-O)^{2}
$$

with respect to the elements of $C$ (coefficients). Here, the square denotes the inner product of $M C-O$ with itself. The solution of this problem is given by [38]

$$
\bar{C}=V W^{-1} U^{\dagger} O,
$$

where $U, V$, and $W$ are the matrices that appear in the SVD decomposition of $M$ [Eq. (1)] and $W^{-1}$ is the MoorePenrose pseudoinverse of $W$ [38-41], which is obtained by taking the transpose of $W$ and replacing the nonzero elements of $W^{T}$ with their inverses, while leaving the zero elements unchanged. ${ }^{2}$ Note that the solution in Eq. (3) exists even if the matrix $M$ is noninvertible. The solution exists, for example, if $M$ is not a square matrix or if $M$ has a vanishing determinant.

The matrix $V^{\dagger}$ takes the elements of $C$ from the original basis of coefficients to the principal-component basis. Each element of $P^{C}=V^{\dagger} C$ is one of the principal components. Similarly, the matrix $U^{\dagger}$ yields the principal components of $O: P^{O}=U^{\dagger} O$. Owing to the phase ambiguities in the SVD in each row in $V^{\dagger}$ and each row of $U^{\dagger}$, each principal component is defined only up to an overall phase. By virtue of the unitarity of $V$ and of $U$, the principal components $P^{C}$ are orthogonal to each other, and the principal components $P^{O}$ are orthogonal to each other. Owing to the fact the $W$ is diagonal, each principal component in $P^{C}$ is coupled to only a single principal component in $P^{O}$, and vice versa.

The best-fit values of the principal components $P^{C}$ are given by the elements of

$$
\bar{P}^{C}=V^{\dagger} \bar{C}=W^{-1} U^{\dagger} O,
$$

where we have used Eq. (3). The fluctuation in $M C$ that is produced by a unit fluctuation in a principal component in $P^{C}$ is given by the corresponding singular value in $W$. Hence, the uncertainties $\Delta P^{C}$ in the best-fit values $\bar{P}^{C}$ are given by the inverses of the diagonal values of $W$ :

$$
\Delta P_{i}^{C}=W_{i i}^{-1}
$$

Because $W$ is diagonal, these uncertainties are uncorrelated. Furthermore, they depend on the uncertainty of only a single principal component in $P^{O}$. The principal components that are ill constrained because of the existence of flat directions correspond to near-vanishing diagonal values of $W$. That is, the SVD sequesters linear combinations of coefficients that are ill constrained because of the presence of flat directions in the coefficient space.

\footnotetext{
${ }^{2}$ Throughout this paper, when we refer to the pseudoinverse of a matrix, we mean the Moore-Penrose pseudoinverse, and we use the -1 power of a noninvertible matrix to denote its MoorePenrose pseudoinverse.
}

\section{B. Application of singular-value decomposition to the fitting of SMEFT coefficients}

The fitting of the SMEFT coefficients is carried out by minimizing the $\chi^{2}$, which is defined by

$$
\chi^{2}=\left(O^{\mathrm{SMEFT}}-O^{\mathrm{exp}}\right)^{T}\left(\sigma^{2}\right)^{-1}\left(O^{\mathrm{SMEFT}}-O^{\exp }\right),
$$

where $O^{\exp }$ is the $N_{\text {obs }}$-dimensional column vector of experimental observables, $O^{\text {SMEFT }}$ is the $N_{\text {obs }}$-dimensional column vector of theoretical predictions for the observables in the SMEFT, $\sigma^{2}$ is the $N_{\text {obs }} \times N_{\text {obs }}$ covariance matrix of experimental and theoretical uncertainties, and $N_{\text {obs }}$ is the number of observables. We decompose the theoretical predictions in the SMEFT into standard-model (SM) contributions and beyond-the-standard-model (BSM) contributions as

$$
O^{\mathrm{SMEFT}}=O^{\mathrm{SM}}+O^{\mathrm{BSM}},
$$

and rewrite $\chi^{2}$ as

$$
\chi^{2}=\left(O^{\mathrm{diff}}-O^{\mathrm{BSM}}\right)^{T}\left(\sigma^{2}\right)^{-1}\left(O^{\mathrm{diff}}-O^{\mathrm{BSM}}\right),
$$

where

$$
O^{\text {diff }}=O^{\exp }-O^{\mathrm{SM}}
$$

Now we wish to put $\chi^{2}$ in Eq. (8) into the linear-leastsquares form. First, since the covariance matrix is symmetric, we can diagonalize it:

$$
U_{\mathrm{cov}}^{-1} \sigma^{2} U_{\mathrm{cov}}=\hat{\sigma}^{2} .
$$

We note that the diagonal matrix $\hat{\sigma}^{2}$ and the unitary matrix $U_{\text {exp }}$ can be found conveniently from the SVD decomposition of $\sigma^{2}$, although other diagonalization methods could also be used. Then, we can write $\chi^{2}$ as

$$
\chi^{2}=\left(O^{\mathrm{diff}}-O^{\mathrm{BSM}}\right)^{T} U_{\mathrm{cov}}\left(\hat{\sigma}^{2}\right)^{-1} U_{\mathrm{cov}}^{-1}\left(O^{\mathrm{diff}}-O^{\mathrm{BSM}}\right) .
$$

Since the diagonal matrix $\hat{\sigma}^{2}$ is positive definite, the quantity $\left(\hat{\sigma}^{2}\right)^{-\frac{1}{2}}$ is well defined. Therefore, we can normalize the observables in the new basis to unit error by writing

$$
\begin{gathered}
\hat{O}^{\mathrm{diff}}=\left(\hat{\sigma}^{2}\right)^{-\frac{1}{2}} U_{\mathrm{cov}}^{-1} O^{\mathrm{diff}}, \\
\hat{O}^{\mathrm{BSM}}=\left(\hat{\sigma}^{2}\right)^{-\frac{1}{2}} U_{\mathrm{cov}}^{-1} O^{\mathrm{BSM}} .
\end{gathered}
$$

Now $\chi^{2}$ has the form

$$
\chi^{2}=\left(\hat{O}^{\mathrm{BSM}}-\hat{O}^{\mathrm{diff}}\right)^{2} .
$$

Since $O^{\mathrm{BSM}}$ is linear in the SMEFT coefficients, we can write it in the form 


$$
O^{\mathrm{BSM}}=H C,
$$

where $C$ is an $N_{\text {coeff }}$-dimensional column vector of SMEFT coefficients, $H$ is an $N_{\text {obs }} \times N_{\text {coeff }}$ matrix, and $N_{\text {coeff }}$ is the number of SMEFT coefficients. It follows that

$$
\hat{O}^{\mathrm{BSM}}=M C,
$$

where

$$
M=\left(\hat{\sigma}^{2}\right)^{-1} U_{\operatorname{cov}}^{-1} H
$$

Hence, in order to constrain the SMEFT coefficients, we minimize

$$
\chi^{2}=\left(M C-\hat{O}^{\mathrm{diff}}\right)^{2},
$$

which is a linear-least-squares problem. As was described in Sec. II A, the solution of this minimization problem can be obtained from the SVD decomposition $M=U W V^{\dagger}$ :

$$
\bar{C}=V W^{-1} U^{\dagger} \hat{O}^{\mathrm{diff}} .
$$

We emphasize that the expression in Eq. (18) provides a convenient way to obtain the values of the coefficients that are well determined, even in the presence of flat directions in the coefficient space. If there are flat directions, then the formal solution of the linear least squares problem ${ }^{3}$

$$
\bar{C}=\left[H^{T}\left(\sigma^{2}\right)^{-1} H\right]^{-1} H^{T}\left(\sigma^{2}\right)^{-1} O_{\text {diff }}
$$

is ill defined because the ordinary inverse of the SMEFT covariance matrix

$$
\sigma_{\text {SMEFT }}^{2}=H^{T}\left(\sigma^{2}\right)^{-1} H
$$

does not exist. However, in Eq. (18), $W^{-1}$ is the MoorePenrose pseudoinverse of $W$ and is well defined, even in the presence of flat directions. Owing to the properties of the pseusdoinverse, the expression in Eq. (18) sets the values of the undetermined coefficients to zero.

As we will demonstrate, the best-fit values of the individual SMEFT coefficients and their uncertainties do not accurately characterize the constraints on the SMEFT coefficients. The principal components in the coefficient space and their uncertainties parametrize the best fit in a much more meaningful form. As we have mentioned, each element of $P^{C}=V^{\dagger} C$ gives one of the principal components of the SMEFT coefficients. The best-fit values of the principal components are given by the elements of $\bar{P}^{C}$ in Eq. (4), and the one-standard-deviation uncertainty on each principal component is given by the corresponding element of $\Delta \bar{P}^{C}$ in Eq. (5).

\footnotetext{
${ }^{3}$ See, for example, Eq. (4.3) of Ref. [25].
}

In terms of the SVD quantities, the covariance matrix of the SMEFT coefficients is obtained by using $V$ to rotate $W$ back to the original basis of SMEFT coefficients:

$$
\sigma_{\text {SMEFT }}^{2}=V W^{-1}\left(W^{-1}\right)^{\dagger} V^{\dagger} .
$$

As is standard, the covariance matrix for the situation in which one has marginalized over some of the coefficients is obtained by striking from the full covariance matrix the rows and columns that correspond to the marginalized coefficients [42]. Although the covariance matrix contains the same information as the uncertainties in the principal components, we will see that the presentation of uncertainties in principal-component form leads to a clearer picture of the constraints on the SMEFT coefficients.

The Fisher information matrix is given by the inverse of the covariance matrix in Eq. (20):

$$
\mathcal{I}=\sigma_{\mathrm{SMEFT}}^{2}=H^{T}\left(\sigma^{2}\right)^{-1} H,
$$

from which it follows, using Eq. (21), that

$$
\mathcal{I}=V W^{\dagger} W V^{\dagger} .
$$

In Refs. [20-25], the principal components of the SMEFT coefficients $\left(P^{C}\right)$ are obtained by diagonalizing the Fisher matrix. We note that the Fisher information is the absolute square of the matrix $M=U W V^{\dagger}$ that is analyzed in the SVD approach. Indeed, one method for implementing SVD involves finding the eigenvectors and eigenvalues of $W$ by diagonalizing the absolute square of $M$. However, it is well known that this method can be imprecise. Other, more precise, methods for carrying out SVD have been devised [27]. The reason for the possible imprecision in diagonalizing the Fisher matrix is that the absolute square of $M$ has a condition number that is equal to the square of the condition number of $M$. If the condition number is very large, as is the case when there are nearly flat directions such that $M$ has eigenvalues that differ greatly in size, then there could, in principle be practical advantages in using SVD, rather than diagonalization of the Fisher matrix, to obtain the principal components.

A conceptual advantage of the SVD method over the Fisher-matrix method is that it relates the principal components of the SMEFT coefficients $\left(P^{C}\right)$ to the principal components of the observables $\left(P^{O}\right)$. Because $W$ is diagonal, the relationship is one-to-one:

$$
P_{i}^{C}=W_{i i} P_{i}^{O} .
$$

The relation in Eq. (24) shows which linear combination of observables affects a given SMEFT-coefficient principal component. This insight could allow one to identify new measurements that could further constrain a poorly constrained SMEFT-coefficient principal component. 
Conversely, the relation in Eq. (24) would allow one to identify a linear combination of observables that would be particularly sensitive to the existence of physics beyond the standard model, as parametrized by a SMEFT-coefficient principal component.

\section{APPLICATION TO TOP-QUARK DECAY}

In this section, we illustrate the PCA/SVD method for fitting the SMEFT coefficients by applying it to the case of top-quark decay to a $b$ quark and a $W$ boson.

\section{A. SMEFT operators}

We work in the Warsaw-basis [1] of SMEFT operators, and our notation is similar to that of Ref. [43]. Following Ref. [31], we fit the coefficients $C_{t W}, C_{b W}, C_{\phi t b}, C_{t g}$, and $C_{b g}$, which correspond to the operators

$$
\begin{aligned}
\mathcal{O}_{t W} & =\mathcal{O}_{b 3}, \\
\mathcal{O}_{b W} & =\mathcal{O}_{33}, \\
\mathcal{O}_{\phi t b} & =\mathcal{O}_{\phi b u}, \\
\mathcal{O}_{t g} & =\mathcal{O}_{33}, \\
\mathcal{O}_{b g} & =\mathcal{O}_{\substack{d g \\
33}}
\end{aligned}
$$

with

$$
\begin{aligned}
\mathcal{O}_{p w} p & =\bar{q}_{p} \sigma^{\mu \nu} u_{r} \tau^{I} \tilde{\phi} W_{\mu \nu}^{I}, \\
\mathcal{O}_{d W} & =\bar{q}_{p} \sigma^{\mu \nu} d_{r} \tau^{I} \phi W_{\mu \nu}^{I}, \\
\mathcal{O}_{p u d} & =i\left(\tilde{\phi}^{\dagger} D_{\mu} \phi\right)\left(\bar{u}_{p} \gamma^{\mu} d_{r}\right), \\
\mathcal{O}_{p q} & =\bar{q}_{p} \sigma^{\mu \nu} T^{A} u_{r} \tilde{\phi} G_{\mu \nu}^{A}, \\
\mathcal{O}_{p q} & =\bar{q}_{p} \sigma^{\mu \nu} T^{A} d_{r} \tilde{\phi} G_{\mu \nu}^{A} .
\end{aligned}
$$

We also consider the coefficients $C_{q q}^{(1)}, C_{q q}^{(3)}, C_{q u}^{(1)}, C_{q u}^{(8)}$, and $C_{l q}^{(3)}$, which correspond to the four-fermion operators

$$
\begin{aligned}
& \mathcal{O}_{q q}^{(1)}=\left(\bar{q}_{p} \gamma^{\mu} q_{r}\right)\left(\bar{q}_{s} \gamma_{\mu} q_{t}\right), \\
& \mathcal{O}_{q q}^{(3)}=\left(\bar{q}_{p} \gamma^{\mu} \tau^{a} q_{r}\right)\left(\bar{q}_{s} \gamma_{\mu} \tau^{a} q_{t}\right), \\
& \mathcal{O}_{q u}^{(1)}=\left(\bar{q}_{p} \gamma^{\mu} q_{r}\right)\left(\bar{u}_{s} \gamma_{\mu} u_{t}\right), \\
& \mathcal{O}_{q u}^{(8)}=\left(\bar{q}_{p} \gamma^{\mu} T^{A} q_{r}\right)\left(\bar{u}_{s} \gamma_{\mu} T^{A} u_{t}\right), \\
& \mathcal{O}_{l q}^{(3)}=\left(\bar{q}_{p} \gamma^{\mu} \tau^{a} q_{r}\right)\left(\bar{q}_{s} \gamma_{\mu} \tau^{a} q_{t}\right) .
\end{aligned}
$$

Here, $q_{p}\left(l_{p}\right)$ is a left-handed quark (lepton) isospin doublet with generation index $p, u_{r}$ and $d_{r}$ are the up and down right-handed isospin singlets with generation index $\mathrm{r}, l_{r}$ is the lepton right-handed isospin singlet with generation index $\mathrm{r}, \phi$ is the Higgs isospin doublet, $\tilde{\phi}=i \tau_{2} \phi^{*}$ is the hypercharge-conjugate Higgs doublet, $\tau$ is a Pauli matrix, $W_{\mu \nu}^{I}$ is the field-strength tensor for the $S U(2)_{I}$ gauge bosons with isospin index $I$, the $\gamma$ 's are Dirac matrices, $\sigma_{\mu \nu}=i\left[\gamma_{\mu}, \gamma_{\nu}\right], G_{\mu \nu}^{A}$ is the gluon field-strength tensor with color index $A$, and $T^{A}$ is a color matrix in the fundamental representation with color index $A$.

\section{B. Experimental inputs}

We take experimental values of the total top-quark decay rate and the helicity fractions from the Particle Data Group compilation [44]:

$$
\begin{aligned}
& \Gamma_{\text {tot }}^{\exp }=1.41_{-0.15}^{+0.19} \mathrm{GeV}, \\
& F_{L}^{\exp }=0.687 \pm 0.018, \\
& F_{-}^{\exp }=0.320 \pm 0.013
\end{aligned}
$$

In our analysis, we symmetrize the uncertainties in $\Gamma_{\text {tot }}^{\text {exp }}$ by shifting the central value. That is, we take $\Gamma_{\text {tot }}^{\exp }=1.43 \pm 0.17 \mathrm{GeV}$. The correlation matrix of the experimental uncertainties is [45]

$$
\rho=\left(\begin{array}{ccc}
1.0 & 0 & 0 \\
0 & 1.0 & -0.87 \\
0 & -0.89 & 1.0
\end{array}\right) .
$$

Then, the experimental inputs for our SVD analysis are $O^{\exp }=\left(\Gamma_{\text {tot }}^{\exp }, F_{L}^{\exp }, F_{-}^{\exp }\right)^{T}$ and $\sigma_{i j}^{2}=\sigma_{i} \rho_{i j} \sigma_{j}$. Here $i, j=1$, 2 , and 3 correspond to $\Gamma_{\text {tot }}^{\exp }, F_{L}^{\exp }$, and $F_{-}^{\exp }$, respectively, and the $\sigma_{i}$ are the experimental uncertainties.

\section{Theoretical inputs}

We make use of the expressions in Ref. [31] for SMEFT contributions to $\Gamma_{\text {tot }}$, the total decay width to $b W, F_{L}$, the fractional decay rate for a longitudinally polarized $W$ boson, and $F_{-}$, the fractional decay rate for a $W$ boson with negative helicity. ${ }^{4}$ We include the QCD corrections that are given in Ref. [31]. We note that the standard-model QCD corrections are also given in Ref. [47] and that an analysis of the SMEFT contributions to $t$ quark decay has also been given in Ref. [48].

For purposes of this demonstration, we do not include uncertainties in the theoretical predictions. They could be incorporated into the analysis by adding the theoretical covariance matrix to the experimental covariance matrix. ${ }^{5}$

The input parameters for the theoretical calculation are given in Table I and are identical to those in Ref. [31], except that we evaluate $\alpha_{s}$ at the scale $m_{t}$, rather than the scale $M_{Z}$. We compute the electroweak coupling $\bar{g}$ from [49]

\footnotetext{
${ }^{4}$ The expressions for these quantities in the published version of Ref. [31] have been corrected in the second arXiv version of that paper [46].

${ }^{5}$ If the theoretical and experimental uncertainties are correlated, say, through the use of a common parameter in the theoretical and experimental analyses, then one would need to construct a complete covariance matrix of theoretical and experimental uncertainties, including entries for that parameter, and then marginalize over that parameter.
} 
TABLE I. Input parameters for the theoretical calculation.

\begin{tabular}{lccc}
\hline \hline$M_{Z}$ & $91.1876 \mathrm{GeV}$ & $M_{W}$ & $80.379 \mathrm{GeV}$ \\
$v$ & $246 \mathrm{GeV}$ & $m_{t}$ & $173.0 \mathrm{GeV}$ \\
$m_{b}$ & $4.78 \mathrm{GeV}$ & $G_{F}$ & $1.1664 \times 10^{-5} \mathrm{GeV}^{-2}$ \\
$\alpha_{\mathrm{em}}^{-1}$ & 137.036 & $\alpha_{s}\left(m_{t}\right)$ & 0.1081 \\
\hline \hline
\end{tabular}

$$
\bar{g}^{2}=2 \sqrt{2} G_{F} M_{Z}^{2}\left(1+\sqrt{1-\frac{4 \pi \alpha_{\mathrm{em}}}{\sqrt{2} G_{F} M_{Z}^{2}}}\right) .
$$

We set the SMEFT cutoff to be $\Lambda=500 \mathrm{GeV}$.

\section{Fit with one SMEFT coefficient}

In Table II, we show the best-fit values of the SMEFT coefficients and their two-standard-deviation uncertainties that are obtained by setting all of the coefficients to zero, except for one. This is a widely used approach for constraining the SMEFT coefficients. However, as we will see, it can be quite misleading.

\section{E. Fit with three SMEFT coefficients}

Now let us consider the case in which only the first three coefficients in Table II are nonzero. Then, we can compute the best-fit values of those coefficients and their uncertainties, marginalized over the other two coefficients. As we explained earlier, the latter can be obtained from the diagonal values of the covariance matrix. The results of this computation are shown in Table III. As can be seen, the central values have shifted substantially relative to those in Table II, and the uncertainties have increased, in some cases by almost an order of magnitude. Clearly, the singlecoefficient values and uncertainties in Table II are not indicative of the true constraints on the SMEFT coefficients

TABLE II. Best-fit values of the SMEFT coefficients and their two-standard-deviation uncertainties computed by setting all of the coefficients to zero, except for one.

\begin{tabular}{lrrr}
\hline \hline$C_{t W}$ & $0.0644 \pm 0.100$ & $C_{q q}^{(1)}$ & $-4.81 \pm 37.1$ \\
$C_{t g}$ & $-3.65 \pm 8.22$ & $C_{q q}^{(3)}$ & $0.656 \pm 5.07$ \\
$C_{b W}$ & $0.679 \pm 1.03$ & $C_{q u}^{(1)}$ & $14.1 \pm 56.4$ \\
$C_{b g}$ & $-13.5 \pm 17.9$ & $C_{q u}^{(8)}$ & $10.6 \pm 42.3$ \\
$C_{\phi t b}$ & $2.94 \pm 5.56$ & $C_{l q}^{(3)}$ & $-4.35 \pm 33.6$ \\
\hline \hline
\end{tabular}

TABLE III. Best-fit values of the SMEFT coefficients obtained by keeping only three coefficients nonzero. The uncertainties are the two-standard-deviation uncertainties that are obtained by marginalizing over two of the coefficients.

\begin{tabular}{lc}
\hline \hline$C_{t W}$ & $0.0209 \pm 0.333$ \\
$C_{t g}$ & $4.73 \pm 62.8$ \\
$C_{b W}$ & $1.13 \pm 8.72$ \\
\hline \hline
\end{tabular}

in the presence of three nonzero coefficients. However, the large uncertainties in Table III paint an unduly pessimistic picture of the constraints that can be achieved.

In order to see this, let us carry out a PCA with three nonzero coefficients. Making use of the SVD method, we obtain

$$
\begin{aligned}
U^{\dagger} & =\left(\begin{array}{ccc}
-0.273 & -0.770 & -0.576 \\
-0.276 & -0.511 & 0.814 \\
0.922 & -0.381 & 0.0732
\end{array}\right), \\
V^{\dagger} & =\left(\begin{array}{ccc}
-0.999 & 0.000697 & -0.0414 \\
0.0411 & 0.136 & -0.990 \\
-0.00496 & 0.991 & 0.136
\end{array}\right),
\end{aligned}
$$

and

$$
W=\left(\begin{array}{ccc}
19.9 & 0 & 0 \\
0 & 1.77 & 0 \\
0 & 0 & 0.0315
\end{array}\right)
$$

Here, and throughout the remainder of this paper, when we present the array $V^{\dagger}$, the columns correspond to the following order of the SMEFT coefficients: $C_{t W}, C_{t g}$, $C_{b W}, C_{b g}, C_{\phi t b}, C_{q q}^{(1)}, C_{q q}^{(3)}, C_{q u}^{(1)}, C_{q u}^{(8)}, C_{l q}^{(3)}$. When we present the array $U^{\dagger}$, the columns correspond to the following order of the experimental observables: $\Gamma_{\text {tot }}^{\exp }$, $F_{L}^{\exp }, F_{-}^{\exp }$.

From the expression for $V^{\dagger}$, we see that the principal components of the SMEFT coefficients are

$$
\begin{aligned}
& P_{1}^{C}=0.999 C_{t W}-0.000697 C_{t g}+0.0414 C_{b W}, \\
& P_{2}^{C}=-0.0411 C_{t W}-0.136 C_{t g}+0.990 C_{b W}, \\
& P_{3}^{C}=-0.00496 C_{t W}+0.991 C_{t g}+0.136 C_{b W} .
\end{aligned}
$$

The best-fit values of the principal components are given by the elements of $\bar{P}^{C}$ in Eq. (4), and their two-standarddeviation uncertainties are given by twice the inverse of the corresponding diagonal element of $W$. The results are

$$
\begin{aligned}
& \bar{P}_{1}^{C}=-0.0645 \pm 0.100 \\
& \bar{P}_{2}^{C}=-0.477 \pm 1.13 \\
& \bar{P}_{3}^{C}=4.84 \pm 63.4
\end{aligned}
$$

We see that $P_{1}^{C}$ and $P_{2}^{C}$ are much better constrained than any of the individual coefficients and that only $P_{3}^{C}$ is poorly constrained. The PCA analysis clearly allows one to access a much more powerful set of constraints than do the analyses of individual SMEFT coefficients.

The principal components of the observables are given by the rows of $U^{\dagger}$ : 


$$
\begin{aligned}
& P_{1}^{O}=-0.273 O_{1}-0.770 O_{2}-0.576 O_{3} \\
& P_{2}^{O}=-0.276 O_{1}-0.511 O_{2}+0.814 O_{3} \\
& P_{3}^{O}=+0.992 O_{1}-0.381 O_{2}+0.0732 O_{3} .
\end{aligned}
$$

As we have mentioned, each observable principal component $P_{i}^{O}$ is coupled through $W$ to a single coefficient principal component $P_{i}^{C}$, and vice versa. From the elements of $W$, we see that $P_{1}^{C}$ is highly constrained by $P_{1}^{O}$ and, conversely, $P_{1}^{O}$ would be very sensitive to new physics, as parametrized by $P_{1}^{C}$. This suggests that one might look for new physics be tightening the experimental bound on $P_{1}^{O}$. On the other hand, $P_{2}^{O}$ is less strongly coupled to $P_{2}^{C}$, and $P_{3}^{O}$ is even less strongly coupled to $P_{3}^{C}$. This suggests that it might be useful to search for additional SMEFT operators that affect the linear combination of obervables in $P_{2}^{O}$ and $P_{3}^{O}$.

\section{F. Fit with five SMEFT coefficients}

Now suppose that we keep only the first five SMEFT coefficients in Table II nonzero. In this case, we have more SMEFT coefficients than observables, and so the individual coefficients cannot be fit unambiguously. Furthermore, because there are necessarily flat directions, the marginalization over some sets of SMEFT coefficients is illdefined. Nevertheless, the PCA/SVD approach allows us to find meaningful constraints. We obtain

$$
\begin{gathered}
U^{\dagger}=\left(\begin{array}{cccc}
-0.273 & -0.770 & -0.576 \\
0.275 & 0.512 & -0.814 \\
-0.922 & 0.381 & -0.0720
\end{array}\right), \quad \text { (35) } \\
V^{\dagger}=\left(\begin{array}{ccccc}
-0.999 & 0.000698 & -0.0414 & 0.00390 & -0.00316 \\
0.0411 & 0.134 & -0.969 & 0.0443 & -0.196 \\
0.00282 & -0.931 & -0.0541 & 0.0222 & -0.361 \\
0.00199 & 0.0163 & 0.0453 & 0.999 & 0.0128 \\
0.00647 & -0.340 & -0.231 & 0.00431 & 0.912
\end{array}\right),
\end{gathered}
$$

and

$$
W=\left(\begin{array}{ccccc}
19.9 & 0 & 0 & 0 & 0 \\
0 & 1.80 & 0 & 0 & 0 \\
0 & 0 & 0.0339 & 0 & 0
\end{array}\right)
$$

The coefficients of the SMEFT coefficients of a given principal component are given by the entries in the corresponding row in $V^{\dagger}$. The best-fit values for the first three principal components and their two-standarddeviation uncertainties are

$$
\begin{aligned}
& \bar{P}_{1}^{C}=-0.0645 \pm 0.100, \\
& \bar{P}_{2}^{C}=-0.467 \pm 1.11, \\
& \bar{P}_{3}^{C}=-4.46 \pm 59.0 .
\end{aligned}
$$

The linear combinations of SMEFT coefficients that are unconstrained have been sequestered by the SVD procedure. Comparing Eq. (38) with Eq. (33), we see that the best-fit values and uncertainties in the first two principal components are remarkably stable as new SMEFT contributions are introduced. This reflects the fact that the observables are relatively insensitive to the SMEFT contributions that are proportional to $C_{b g}$ and $C_{\phi t b}$, as can be seen from the small coefficients of $C_{b g}$ and $C_{\phi t b}$ in the first two principal components. We see that the principal components of the observables, which are given by the rows of $U^{\dagger}$, are almost unchanged in comparison with those from the three-coefficient fit.

\section{G. Fit with ten SMEFT coefficients}

Next we apply the SVD method to the complete set of ten SMEFT coefficients in Table II. We list only the three principal components that are constrained. They are

$$
\begin{aligned}
P_{1}^{C}= & -0.999 C_{t W}+0.000698 C_{t g}-0.0414 C_{b W}+0.00390 C_{b g}-0.00316 C_{\phi t b} \\
& +0.000737 C_{q q}^{(1)}-0.00540 C_{q q}^{(3)}-0.000570 C_{q u}^{(1)}-0.000760 C_{q u}^{(8)}+0.000815 C_{l q}^{(3)}, \\
P_{2}^{C}= & 0.0406 C_{t W}+0.134 C_{t g}-0.967 C_{b W}+0.0442 C_{b g}-0.196 C_{\phi t b} \\
& -0.00856 C_{q q}^{(1)}+0.0628 C_{q q}^{(3)}-0.00550 C_{q u}^{(1)}-0.00733 C_{q u}^{(8)}-0.00947 C_{l q}^{(3)}, \\
P_{3}^{C}= & 0.00819 C_{t W}-0.0753 C_{t g}-0.0672 C_{b W}+0.00484 C_{b g}-0.0452 C_{\phi t b} \\
& +0.131 C_{q q}^{(1)}-0.964 C_{q q}^{(3)}+0.0854 C_{q u}^{(1)}+0.114 C_{q u}^{(8)}+0.145 C_{l q}^{(3)} .
\end{aligned}
$$

The best-fit values and two-standard-deviation uncertainties for these principal components are 


$$
\begin{aligned}
& \bar{P}_{1}^{C}=-0.0645 \pm 0.100, \\
& \bar{P}_{2}^{C}=-0.465 \pm 1.11, \\
& \bar{P}_{3}^{C}=-0.432 \pm 5.32 .
\end{aligned}
$$

As can be seen, the first two principal components remain quite stable in best-fit value and uncertainty as additional SMEFT coefficients are introduced, reflecting the relative insensitivity of the observables to the additional SMEFT coefficients.

The principal components of the observables can be obtained from $U^{\dagger}$, which is given by

$$
U^{\dagger}=\left(\begin{array}{ccc}
-0.273 & -0.770 & -0.576 \\
0.287 & 0.507 & -0.813 \\
-0.918 & 0.387 & -0.0829
\end{array}\right)
$$

This result for $U^{\dagger}$ is not very different from the results from the three- or five-coefficient fits.

The results in Eq. (40) express precisely the constraints on the 10-coefficient fit that follow from the input topquark decay rates. As such, they should be considered to supersede the results from the one- and two-coefficient fits that were given in Ref. [31], since those fits fail to account for the high degree of correlation between uncertainties in the SMEFT coefficients, and, consequently, are quite misleading.

\section{H. Fit with a flat direction in coefficient space}

Next, we examine the case in which the number of SMEFT coefficients and the number of observables are equal, but there is a hidden flat direction. In order to construct an example of this situation, we keep three SMEFT coefficients, $C_{t W}, C_{t g}$, and $C_{b W}$, nonzero and set the remaining SMEFT coefficients to zero. Let $a_{t W}, a_{t g}$, and $a_{b W}$ be the coefficients of $C_{t W}, C_{t g}$, and $C_{b W}$ in $O^{\text {SMEFT }}$. Then, the following replacement creates an approximate artificial flat direction in the space of $C_{t g}$ and $C_{b W}$ :

$$
a_{t g} \rightarrow \epsilon a_{t g}+(1-\epsilon) r a_{b W} .
$$

In the limit $\epsilon \rightarrow 0$, there is an exact flat direction in the space of SMEFT coefficients.

As a numerical example, we take scale factor $r$ in Eq. (42) to be -3.2 . As $\epsilon$ approaches zero, conventional fitting procedures that use gradients of $\chi^{2}$ to find a minimum in $\chi^{2}$ have numerical difficulties. Let us consider, for example, the situation for $\epsilon=10^{-6}$. The Mathematica routine FindMinimum can be used to minimize the $\chi^{2}$. This routine comes with a number of options for the method to be used in finding the minimum. Using Mathematica version 11.3 [34], we find that the conjugate-gradient method algorithm yields

$$
\begin{aligned}
C_{t W} & =0.0445, \\
C_{t g} & =0.236, \\
C_{b W} & =1.24,
\end{aligned}
$$

Newton's method yields

$$
\begin{aligned}
C_{t W} & =0.0410, \\
C_{t g} & =7.00 \times 10^{5}, \\
C_{b W} & =2.24 \times 10^{6},
\end{aligned}
$$

and the principal-axis method yields

$$
\begin{aligned}
C_{t W} & =0.0445, \\
C_{t g} & =0.161, \\
C_{b W} & =1.00 .
\end{aligned}
$$

Owing to the existence of a nearly flat direction, the results vary wildly, depending on the algorithm that is used in minimizing $\chi^{2}$. While this behavior is to be expected in under-determined problems, it is difficult to draw any conclusion from such results. In contrast, meaningful constraints can be found by using PCA. From SVD, we obtain

$V^{\dagger}=\left(\begin{array}{ccc}-0.988845 & 0.142167 & -0.0444274 \\ -0.148947 & -0.943833 & 0.294948 \\ -1.49286 \times 10^{-9} & 0.298275 & 0.95448\end{array}\right)$

and

$$
W=\left(\begin{array}{ccc}
20.1307 & 0 & 0 \\
0 & 5.80765 & 0 \\
0 & 0 & 9.49318 \times 10^{-9}
\end{array}\right) .
$$

The coefficient principal components are

$P_{1}^{C}=-0.989 C_{t W}+0.142 C_{t g}-0.0444 C_{b W}$,

$P_{2}^{C}=-0.149 C_{t W}-0.944 C_{t g}+0.295 C_{b W}$,

$P_{3}^{C}=-1.49 \times 10^{-9} C_{t W}+0.298 C_{t g}+0.954 C_{b W}$.

From the result for $W$, it follows that only $P_{1}^{C}$ and $P_{2}^{C}$ are well constrained, while $P_{3}^{C}$ is not, as is evident from the near-vanishing of the corresponding diagonal value of $W{ }^{6}$ The observable principal component $P_{3}^{O}$ has essentially no sensitivity to the physics beyond the standard model that is

\footnotetext{
${ }^{6}$ We keep more significant digits than usual in the results for $V^{\dagger}$ and $W$ in Eqs. (46) and (47) for purposes of later comparison with the results for $V^{\dagger}$ and $W$ when $\epsilon=0$.
} 
embodied in $P_{3}^{C}$. We see that the SVD/PCA method has constrained the principal components that contain a contribution that is proportional to $-3.2 C_{t g}+C_{b W}$ and has identified as unconstrained the principal component that contains a contribution that is proportional to $C_{t g}+3.2 C_{b W}$, which corresponds to the flat direction. The best-fit values of the principal components and their two-standard deviation uncertainties are

$$
\begin{aligned}
& \bar{P}_{1}^{C}=-0.0655 \pm 0.0994, \\
& \bar{P}_{2}^{C}=0.136 \pm 0.344, \\
& \bar{P}_{3}^{C}=1.58 \times 10^{7} \pm 2.11 \times 10^{8} .
\end{aligned}
$$

We can invert the relations in Eq. (48), using the rows of $V$ to obtain the coefficients. The result is

$$
\begin{aligned}
C_{t W} & =-0.989 P_{1}^{C}-0.149 P_{2}^{C}-1.49 \times 10^{-9} P_{3}^{C}, \\
C_{t g} & =0.142 P_{1}^{C}-0.944 P_{2}^{C}+0.298 P_{3}^{C}, \\
C_{b W} & =-0.0444 P_{1}^{C}+0.295 P_{2}^{C}+0.954 P_{3}^{C},
\end{aligned}
$$

From Eq. (50), it is easily seen that, to good approximation, the differences between the three results from FindMinimum correspond to differences in the value of $P_{3}^{C}$.

When $\epsilon=0$ and there is an exact flat direction, numerical minimization of $\chi^{2}$ with respect to the SMEFT coefficients would fail to converge to a result. However, the SVD method still yields meaningful constraints. Specifically, we have

$$
V^{\dagger}=\left(\begin{array}{ccc}
-0.988845 & 0.142168 & -0.0444274 \\
-0.148948 & -0.943833 & 0.294948 \\
1.29707 \times 10^{-17} & 0.298275 & 0.95448
\end{array}\right)
$$

and

$$
W=\left(\begin{array}{ccc}
20.1307 & 0 & 0 \\
0 & 5.80765 & 0 \\
0 & 0 & 0
\end{array}\right)
$$

which are very close to the results for $\epsilon=10^{-6}$.

\section{Comparison of SVD with diagonalization of the Fisher matrix}

Finally, let us compare the speed and accuracy of the computation of the eigenvalues and eigenvectors of the Fisher matrix through direct calculation and through the use of SVD. We consider the case of ten SMEFT coefficients, which is the most challenging computationally. In that case, when we evaluate expressions in Mathematica 12 [50], the direct-diagonalization and SVD methods lead to eigenvalues of $W$ (square roots of the eigenvalues of the Fisher matrix) whose relative differences are no more than $4 \times 10^{-16}$ and eigenvectors whose nonzero components have relative differences that are no more than $6.8 \times 10^{-14}$. Clearly, these are insignificant in comparison with other uncertainties in the fits, although they might become more significant in fitting programs that use single-precision arithmetic. Differences in computation time are also insignificant for matrices of this size, as computation times for both methods are on the order of $10^{-5}$ seconds on a modern laptop.

In the case of three nonzero coefficients with an artificial nearly flat direction, the accuracy of the smallest eigenvalue suffers in the case of the Fisher-matrix method, as it becomes negative $\left(-7 \times 10^{-15}\right.$ for the eigenvalue of the Fisher matrix versus $9 \times 10^{-9}$ for the eigenvalue of $W$ ). However, this unphysical result that arises from the Fishermatrix method has no practical consequences for a SMEFT analysis because large excursions of SMEFT coefficients from zero can be bounded by appealing to power counting arguments [20].

The issue of computation time might become more significant in the analysis of larger matrices, such as those that would appear in a full fit of the dimension-8 SMEFT coefficients. In particular, if the number of SMEFT coefficients is much greater than the number of observables, then the Fisher matrix is much larger than the matrix that is analyzed in SVD. In this situation, the computation time for an SVD analysis can be much less than for diagonalization of the Fisher matrix. For example, if one takes $M$ to be a random matrix $100 \times 3000$ matrix, then the computation time to find eigenvalues and eigenfunctions is about 1 second for SVD and 11 seconds for the Fishermatrix method in Mathematica 12 on a modern laptop. These computation-time differences would be magnified if many fits need be performed, for example, in varying input parameters or in using the iterative approach that is described in Sec. IV. This computational advantage of the SVD method over the Fisher-matrix method arises only when the number of SMEFT coefficients is much greater than the number of observables, and, in fact, the Fishermatrix method has the computational advantage when the situation is reversed and the number of observables is much greater than the number of SMEFT coefficients. That advantage would be significant only when the number of observables is very large $(\gtrsim 1000)$.

\section{EXTENSION TO HIGHER ORDERS IN THE SMEFT EXPANSION}

The SVD method that we have presented is limited to fitting problems in which the observables depend linearly on SMEFT coefficients. This is the case for fits at the leading nontrivial order in the effective-field-theory expansion, in which one considers only the contributions of the interference of the dimension-6 SMEFT-operator 
amplitudes with the dimension-4 standard-model-operator amplitudes. At the next order in the SMEFT expansion, one would need to consider not only the contribution from the square of the dimension-6 SMEFT-operator amplitudes, but also the interference of the dimension- 4 standard-modeloperator amplitudes with the thousands of dimension-8 SMEFT-operator amplitudes-a task that is not likely to be undertaken soon.

Nevertheless, beyond leading order, one might still apply the SVD method by making use of an iterative procedure. One could first carry out a fit that retains only the interference of the dimension- 6 amplitude with the dimension- 4 amplitude. Then, one could compute the contribution of the square of the dimension- 6 amplitude, subtract it from the experimental values of the observables, and carry out a new fit, including the dimension- 8 operators. This last step could be iterated to produce fits of the desired accuracy. The iteration process should converge if the effective-field-theory expansion is valid, that is, if the square of the dimension- 6 amplitude is less than the interference of the dimension- 6 amplitude with the dimension- 4 amplitude.

This method would yield best-fit values of the coefficients, but would not give accurate results for the principal components. Instead, one could compute the principal components as follows. First one could obtain the Fischer matrix (inverse covariance matrix) by computing analytically two derivatives of $\chi^{2}$ with respect to the SMEFT coefficients and evaluating the result at the bestfit values of the coefficients from the iterative procedure. The Fischer matrix could be diagonalized by standard methods, and the principal components could then be obtained from the elements of the unitary transformation that effects the diagonalization. The uncertainties would be given by the inverses of the square roots of the diagonal components of the Fischer matrix. Although the Fischermatrix method is used in the last step, this step occurs only once in the procedure. The "heavy-lifting," involving the repeated, iterative principal-component analysis of large matrices, can be carried out by making use of SVD.

\section{SUMMARY}

In using experimental data to constrain the Wilson coefficients in standard model effective field theory (SMEFT), a difficulty that often arises is that observables may be insensitive to certain linear combinations of SMEFT coefficients. That is, there may be "flat directions" in the space of SMEFT coefficients. This difficulty can arise because, in a partial analysis that is restricted to a particular set of physical processes, the number of experimental observables may be less than the number of SMEFT coefficients. In this case, it is clear that some linear combinations of SMEFT coefficients would not be constrained. However, it can happen that some linear combinations of SMEFT coefficients are poorly constrained even when the number of observables is equal to or greater than the number of SMEFT coefficients to be fit.

A standard approach for dealing with this difficulty is to carry out a principal-component analysis (PCA) of the SMEFT coefficients by diagonalizing the Fisher information matrix [20-25]. In this paper, we have presented an alternative approach for carrying out the PCA that is based on singular-value decomposition (SVD). As we have shown, the SVD method provides information about the sensitivity of experimental observables to SMEFT coefficients that is not accessible in the Fisher-matrix method. That information could be used to identify new measurements that could improve the constraint on a poorly constrained SMEFT-coefficient principal component. It could also be used to target particular linear combinations of observables in searches for new physics.

In principle, SVD may offer superior precision in comparison with diagonalization of the Fisher matrix because SVD involves the analysis of a matrix that is better conditioned than the Fisher matrix. The condition number of the Fisher matrix is the square of the condition number of the matrix that is analyzed in the SVD method, and it may become large if there are nearly flat directions in the space of SMEFT coefficients. In practice, very small eigenvalues of the Fisher that are associated with nearly flat directions are of little physical consequence because the excursions of the corresponding coefficient eigenvectors are limited by SMEFT power-counting arguments [20].

In the situation in which the number of SMEFT coefficients is very large and the number of observables is much smaller, as might occur in an analysis of dimension-8 SMEFT coefficients, the matrix that is analyzed in the SVD method is much smaller than the Fisher matrix, and the SVD method may accrue advantages in computational speed.

As we have pointed out, the SVD method also provides a convenient way to compute the central values of the SMEFT coefficients for constrained directions, even in the presence of flat directions.

We have demonstrated the application of the SVD method to the process of top-quark decay to a $W$ boson and a $b$ quark. In this demonstration, we give specific illustrations of the pitfalls in two widely used fitting approaches, namely, (1) setting all of the coefficients to zero except for one and (2) marginalizing over all of the coefficients except for one. We show that approach (1) leads to shifted central values of the coefficients and underestimated uncertainties, while approach (2) leads to an overly pessimistic assessment of uncertainties, which can be ameliorated through the use of PCA. Our fit for the case of ten SMEFT coefficients should be considered to supersede both the one- and two-coefficient fits in Ref. [31], which do not account for the highly-correlated uncertainties in the SMEFT coefficients. 
In the example of top-quark decay to a $W$ boson and a $b$ quark, the matrices involved are of too small to reveal the putative computational advantages of the SVD method. However, the advantages might become significant in fits involving the many dimension-8 SMEFT coefficients if multiple fits were needed, for example, in varying input parameters or in using the iterative fitting procedure that we have described.

Although the method that we have presented is limited to the case in which the observables depend linearly on the SMEFT coefficients, we have outlined in Sec. IV an iterative extension of the method that can be applied to the nonlinear situation, provided that the contributions of the nonlinear terms to the observables are small in comparison with the contributions of the linear terms. This is the case if the SMEFT expansion converges.

Finally, the method that we have presented relies on a Gaussian probability analysis. While one might ultimately want to improve on a Gaussian approach, it should certainly be adequate for the purpose of carrying out exploratory studies in SMEFT.

\section{ACKNOWLEDGMENTS}

We are grateful to Christopher Murphy for pointing out to us Ref. [26], as well as several recent papers on global fitting of SMEFT coefficients. We thank Radja Boughezal, Chien-Yi Chen, Frank Petriello, and Daniel Wiegand for providing us with a version of Ref. [31] that contains corrected expressions for the total width and helicity fractions in top-quark decay to a $b$ quark and a $W$ boson. We also thank Xiang-peng Wang for checking some of our Mathematica expressions. The work of G. T. B. is supported by the U.S. Department of Energy, Division of High Energy Physics, under Contract No. DE-AC0206CH11357. The work of H.S.C. is funded by the Deutsche Forschungsgemeinschaft (DFG, German Research Foundation) under Germany's Excellence Strategy-EXC-2094-390783311. The submitted manuscript has been created in part by University of Chicago Argonne, LLC, Operator of Argonne National Laboratory. Argonne, a U.S. Department of Energy Office of Science laboratory, is operated under Contract No. DE-AC0206CH11357. The U.S. Government retains for itself, and others acting on its behalf, a paid-up nonexclusive, irrevocable worldwide license in said article to reproduce, prepare derivative works, distribute copies to the public, and perform publicly and display publicly, by or on behalf of the Government.
[1] W. Buchmuller and D. Wyler, Nucl. Phys. B268, 621 (1986).

[2] K. Hagiwara, S. Ishihara, R. Szalapski, and D. Zeppenfeld, Phys. Rev. D 48, 2182 (1993).

[3] G. F. Giudice, C. Grojean, A. Pomarol, and R. Rattazzi, J. High Energy Phys. 06 (2007) 045.

[4] B. Grzadkowski, M. Iskrzynski, M. Misiak, and J. Rosiek, J. High Energy Phys. 10 (2010) 085.

[5] Z. Han and W. Skiba, Phys. Rev. D 71, 075009 (2005).

[6] A. Pomarol and F. Riva, J. High Energy Phys. 01 (2014) 151.

[7] C. Y. Chen, S. Dawson, and C. Zhang, Phys. Rev. D 89, 015016 (2014).

[8] J. Ellis, V. Sanz, and T. You, J. High Energy Phys. 07 (2014) 036.

[9] J. D. Wells and Z. Zhang, Phys. Rev. D 90, 033006 (2014).

[10] A. Falkowski and F. Riva, J. High Energy Phys. 02 (2015) 039.

[11] J. de Blas, M. Ciuchini, E. Franco, S. Mishima, M. Pierini, L. Reina, and L. Silvestrini, Proc. Sci., EPS-HEP2017 (2017) 467 [arXiv:1710.05402].

[12] J. de Blas, O. Eberhardt, and C. Krause, J. High Energy Phys. 07 (2018) 048.

[13] J. Ellis, C. W. Murphy, V. Sanz, and T. You, J. High Energy Phys. 06 (2018) 146.
[14] J. Aebischer, J. Kumar, P. Stangl, and D. M. Straub, Eur. Phys. J. C 79, 509 (2019).

[15] E. da Silva Almeida, A. Alves, N. Rosa Agostinho, O. J. P. Éboli, and M. C. Gonzalez-Garcia, Phys. Rev. D 99, 033001 (2019).

[16] A. Biekötter, T. Corbett, and T. Plehn, SciPost Phys. 6, 064 (2019).

[17] N. P. Hartland, F. Maltoni, E. R. Nocera, J. Rojo, E. Slade, E. Vryonidou, and C. Zhang, J. High Energy Phys. 04 (2019) 100.

[18] M. Cepeda et al., Report from Working Group 2: Higgs Physics at the HL-LHC and HE-LHC, CERN Yellow Rep. Monogr. 7, 221 (2019).

[19] I. Brivio, S. Bruggisser, F. Maltoni, R. Moutafis, T. Plehn, E. Vryonidou, S. Westhoff, and C. Zhang, J. High Energy Phys. 02 (2020) 131.

[20] L. Berthier and M. Trott, J. High Energy Phys. 02 (2016) 069.

[21] L. Berthier, M. Bjørn, and M. Trott, J. High Energy Phys. 09 (2016) 157.

[22] I. Brivio and M. Trott, J. High Energy Phys. 07 (2017) 148; 05 (2018) 136(A).

[23] J. Brehmer, F. Kling, T. Plehn, and T. M. P. Tait, Phys. Rev. D 97, 095017 (2018).

[24] J. Brehmer, F. Kling, I. Espejo, and K. Cranmer, Comput. Software Big Sci. 4, 3 (2020). 
[25] R. Aoude, T. Hurth, S. Renner, and W. Shepherd, arXiv:2003.05432.

[26] C. W. Murphy, Phys. Rev. D 97, 015007 (2018).

[27] See, for example, D. Kalman, College Math. J. 27, 2 (1996).

[28] A. Buckley, C. Englert, J. Ferrando, D. J. Miller, L. Moore, M. Russell, and C.D. White, J. High Energy Phys. 04 (2016) 015.

[29] V. Cirigliano, W. Dekens, J. de Vries, and E. Mereghetti, Phys. Rev. D 94, 034031 (2016).

[30] J. A. Aguilar-Saavedra et al., arXiv:1802.07237.

[31] R. Boughezal, C. Y. Chen, F. Petriello, and D. Wiegand, Phys. Rev. D 100, 056023 (2019).

[32] G. T. Bodwin, H. S. Chung, and J. Repond, J. Instrum. 14, P07002 (2019).

[33] See, for example, C. L. Lawson and R. J. Hanson, Solving Least Squares Problems (Society for Industrial and Applied Mathematics, Philadelphia, 1995).

[34] Wolfram Research, Inc., Mathematica, version 11.3 (Wolfram Research, Inc., Champaign, IL, 2018).

[35] W. H. Press, S. A. Teukolsky, W. T. Vetterling, and B. P. Flannery, Numerical Recipes in $C$ (Cambridge University Press, Cambridge, England, 2002).

[36] S. van der Walt, S. C. Colbert, and G. Varoquaux, Comput. Sci. Eng. 13, 22 (2011).
[37] M. Galassi et al., GNU Scientific Library Reference Manual, 3rd ed. (Network Theory Ltd., 2009), http:// www.gnu.org/software/gsl/.

[38] A. Bjerhammar, Trans. R. Inst. Tech. Stockholm 49, 1 (1951).

[39] E. H. Moore, Bull. Am. Math. Soc. 26, 394 (1920).

[40] R. Penrose, Proc. Cambridge Philos. Soc. 51, 406 (1955).

[41] R. Penrose, Proc. Cambridge Philos. Soc. 52, 17 (1956).

[42] T. B. Schön and F. Lindsten, Manipulating the multivariate Gaussian density, Technical Report No. TR3, Division of Automatic Control, Linköping University, 2011.

[43] A. Dedes, W. Materkowska, M. Paraskevas, J. Rosiek, and K. Suxho, J. High Energy Phys. 06 (2017) 143.

[44] M. Tanabashi et al. (Particle Data Group), Phys. Rev. D 98, 030001 (2018).

[45] V. Khachatryan et al. (CMS Collaboration), Phys. Lett. B 762, 512 (2016).

[46] D. Wiegand (private communication).

[47] M. Fischer, S. Groote, J. G. Korner, and M. C. Mauser, Phys. Rev. D 63, 031501 (2001).

[48] C. Zhang, Phys. Rev. D 90, 014008 (2014).

[49] S. Dawson and P. P. Giardino, Phys. Rev. D 101, 013001 (2020)

[50] Wolfram Research, Inc., Mathematica, version 12.1 (Wolfram Research, Inc., Champaign, IL, 2020). 